\title{
Ultrafast coherent control of giant oscillating molecular dipoles in the presence of static electric fields
}

\author{
Bo Y. Chang, ${ }^{1}$ Seokmin Shin, ${ }^{1}$ Alicia Palacios, ${ }^{2}$ Fernando Martín,, 3 \\ and Ignacio R. Sola ${ }^{4, a)}$ \\ ${ }^{1}$ School of Chemistry (BK21), Seoul National University, Seoul 151-747, South Korea \\ ${ }^{2}$ Departamento de Química, Módulo 13, Universidad Autónoma de Madrid, 28049 Madrid, Spain \\ ${ }^{3}$ Instituto Madrileño de Estudios Avanzados en Nanociencia (IMDEA-Nanociencia), Cantoblanco, \\ 28049 Madrid, Spain \\ ${ }^{4}$ Departamento de Química Física, Universidad Complutense, 28040 Madrid, Spain
}

(Received 25 June 2013; accepted 6 August 2013; published online 23 August 2013)

\begin{abstract}
We propose a scheme to generate electric dipole moments in homonuclear molecular cations by creating, with an ultrashort pump pulse, a quantum superposition of vibrational states on electronic states strongly perturbed by very strong static electric fields. By field-induced molecular stabilization, the dipoles can reach values as large as 50 Debyes and oscillate on a time-scale comparable to that of the slow vibrational motion. We show that both the electric field and the pump pulse parameters can be used to control the amplitude and period of the oscillation, while preventing the molecule from ionizing or dissociating. (C) 2013 AIP Publishing LLC. [http://dx.doi.org/10.1063/1.4818878]
\end{abstract}

\section{INTRODUCTION}

Molecular dynamics is associated to quantum superpositions of Hamiltonian eigenstates. While the nuclear wave function encodes the molecular entity, given by the shape or the geometry, the electronic distribution is responsible for the chemical properties. The most studied dynamical processes in molecules involve superposition of vibrational states belonging to the same electronic state. This is the realm of femtochemistry, which aims at manipulating the nuclear arrangements. Despite the large variety of applications relying in the control of molecular dynamics at the femtosecond time scale, these do not allow one to get complete control of the chemical properties of molecules.

The latter control rather lies in the frontier of attophysics, which was introduced with the prospect of actual manipulation of electronic motion in atoms and molecules. ${ }^{1-3}$ The large energy bandwidths of attosecond pulses give access to the necessary superposition of several electronic states in a molecule. In the present work, we use ultrashort laser pulses to prepare wave packets containing a quantum superposition of both electronic and nuclear degrees of freedom, such that the coupled motion of electrons and nuclei leads to physical phenomena that are observable in the time-scale of the nuclear motion. More specifically, we use the molecular hydrogen ion as benchmark to create large oscillating dipole moments, typically of the order of 10-50 Debyes, whose amplitude and period can be tailored by varying the magnitude of an intense static electric field. This has potential applications in controlling the reactivity of a molecule, which is strongly dependent on its electronic density, and in generating electromagnetic radiation at specific frequencies.

Creation and manipulation of electric dipoles in molecular quantum systems has been previously explored through

$\overline{{ }^{a} \text { Electronic mail: isola@quim.ucm.es }}$ different approaches. For example, quantum information and ultracold chemistry sciences ${ }^{4}$ have devoted significant experimental and theoretical efforts to create and control permanent electric dipoles not only in heteronuclear diatomic molecules ${ }^{4-7}$ but also in homonuclear diatomic molecules. ${ }^{8}$ In the latter work, $\mathrm{Rb}$ dimers with permanent dipole moments were obtained from the binding of ground and Rydberg atomic $\mathrm{Rb}$ states. Such a binding leads to paritysymmetry breaking, hence to charge-localized states, which is the necessary condition for the existence of a permanent dipole. Furthermore, control on electron localization in homonuclear molecules has also been achieved by using ultrashort laser pulses ${ }^{9-12}$ and intense laser fields. ${ }^{13,14} \mathrm{In}$ these works, the ultrashort laser radiation creates and drives the superposition of ionizing states of different parity thus leading to asymmetric electronic densities as the molecule dissociates. ${ }^{15-17}$

Here we also use ultrashort pulses to create the initial quantum superposition of states, but the control mechanism is based on the specific properties of the dipole couplings induced by an external DC field $\mathcal{E}_{0}$ on the $\mathrm{H}_{2}^{+}$molecular ion. ${ }^{13,14,18}$ As the internuclear separation between the atoms, $R$, increases, the dipole coupling between electronic states that dissociate on the same products behaves as $\sim R$. This property was exploited by, e.g., Corkum et al. to "freeze" the nuclear motion by means of strong laser fields, ${ }^{19}$ as well as in other theoretical works using oriented $\mathrm{H}_{2}^{+}$molecules and inducing a beating of the localized electronic density from one proton to the other. ${ }^{13,20}$ Our proposal goes a step further, since we induce a correlated electronic and nuclear motion in $\mathrm{H}_{2}^{+}$that efficiently locates the electron in the vicinity of one the protons - and always the same proton - as the bond stretches and shrinks. The wave packet is initially generated by interaction with an ultrashort pulse although, at variance with previous works, we use a strong static field to control the molecular potentials. 


\section{HAMILTONIAN MODEL: DRESSED STATES OF THE MOLECULE IN THE FIELD}

To track the wave packet dynamics, we use a theoretical method that solves the time-dependent Schrödinger equation (TDSE) for the electron and nuclear motion under the presence of an external field $\mathcal{E}(t)$,

$$
\begin{aligned}
i \frac{\partial}{\partial t} \Psi(z, R, t)= & {\left[-\frac{1}{2 \mu_{e}} \frac{\partial^{2}}{\partial z^{2}}-\frac{1}{M} \frac{\partial^{2}}{\partial R^{2}}-\frac{1}{\sqrt{\left(z-\frac{R}{2}\right)^{2}+1}}\right.} \\
& \left.-\frac{1}{\sqrt{\left(z+\frac{R}{2}\right)^{2}+1}}+\frac{1}{R}+q_{e} z \mathcal{E}(t)\right] \Psi(z, R, t),
\end{aligned}
$$

where $z$ is the electron coordinate, $R$ is the internuclear distance, $M$ is the mass of the proton, $\mu_{e}=2 M /(2 M+1) \approx 1$ is the reduced mass of the electron, and $q_{e}=(2 M+2) /(2 M$ $+1) \approx 1$. It should be noted that this TDSE includes all nonadiabatic couplings (i.e., no Born-Oppenheimer approximation is used) although the electron is forced to move along a line defined by the bond axis through the approximated softcore Coulomb potential. Soft-core potentials allow to reproduce the most important features of the quantum dynamics of diatomic molecules, particularly in conditions of tunneling ionization (Keldysh parameter $\gamma \ll 1$ ), without explicitly considering all the dimensions of the system. ${ }^{21-24}$ The TDSE is solved using the split-operator technique with FFT, ${ }^{25,26}$ in a grid of 1024 points for the nuclear coordinate, and 512 points for the electronic coordinate.

The mechanism of the proposed scheme can be easily explained by looking at the molecular field-induced potentials (FIPs). They were first introduced to describe the dynamics of molecules in the presence of strong laser pulses, as avoided multi-photon crossings, ${ }^{27,28}$ bond softening, ${ }^{29,30}$ and bond hardening ${ }^{31,32}$ among other interesting strong field effects. In the regime of optical pulses, they are often regarded as light-induced potentials. The FIPs are the corresponding adiabatic states in the presence of a strong $D C$ field, $\mathcal{E}_{0}$. Since the field is switched on at all times, they are even more necessary to understand the nuclear dynamics.

First, we obtain the Born-Oppenheimer potentials and the corresponding electronic states $\psi_{j}^{B O}(z ; R)$ from the diagonalization of the one-dimensional $\mathrm{H}_{2}^{+}$electronic Hamiltonian $\mathrm{H}^{\mathrm{BO}}$ at each value of $R, \mathrm{H}^{\mathrm{BO}} \psi_{\mathrm{j}}^{\mathrm{BO}}(z ; R)=\mathrm{V}_{\mathrm{j}}(\mathrm{R}) \psi_{\mathrm{j}}^{\mathrm{BO}}(z ; R)$ (the Hamiltonian in Eq. (1) without the derivatives on $R$ and the field switched off), by using the Fourier Grid Hamiltonian (FGH) method. ${ }^{33}$ Special care was taken to avoid aleatory sign changes in the eigenvectors when the diagonalization is carried out at different values of $R$. By using the calculated $\psi_{j}^{B O}(z ; R)$ wave functions, we also evaluated the dipole coupling $\mu_{i j}(R)=\left\langle\psi_{i}^{B O}(z ; R)|z| \psi_{j}^{B O}(z ; R)\right\rangle_{z}$, (integrated over the electronic coordinate $z$ ).

Accurate $U_{j}(R)$ FIPs and $\psi_{j}^{D S}\left(z ; R, \mathcal{E}_{0}\right)$ can then be computed by diagonalizing the dressed Hamiltonian, $\mathrm{H}^{D S}$, given by the sum of the field free electronic Hamiltonian and the potential describing the interaction with the $D C$ field in the complete basis of bound and continuum $B O$ states. In general, this is a challenging problem because the exact dressed states are not square-integrable and the FIPs are associated to resonances. ${ }^{34,35}$ Since we will only use the FIPs to provide a qualitative understanding of the dynamics resulting from the accurate TDSE calculations, and this dynamics is mainly due to the lowest bound dressed states (as we will show below, ionization and dissociation are negligible in the present scheme), it is appropriate to approximately diagonalize $\mathrm{H}^{D S}$ in a truncated basis only including the 4 lowest $B O$ states.

Thus, we first calculate the FIPs at each internuclear distance, by diagonalizing the nuclear Hamiltonian matrix $H_{\text {nuc }}=T+V_{\text {int }}$, where $T$ is the nuclear kinetic energy matrix and $\mathrm{V}_{\text {int }}$ is the potential energy, formed by the electronic potentials as the diagonal terms $V_{j}^{B O}(R)$, and the transient dipole couplings $\mu_{j k}(R) \mathcal{E}_{0}$ as the off-diagonal elements. In the absence of field-induced conical intersections, the diagonalized Hamiltonian is $\mathrm{H}_{a d} \approx \mathrm{T}+\mathrm{U}$, with the diagonal $\mathrm{U}\left(R, \mathcal{E}_{0}\right)=\mathrm{W}\left(R, \mathcal{E}_{0}\right) \mathrm{V}_{\text {int }} \mathrm{W}^{-1}\left(R, \mathcal{E}_{0}\right)$ giving the approximated FIPs.

In Fig. 1, the dotted lines correspond to the potential energy curves of the isolated target (that will be labeled as $V_{1}$ and $\left.V_{2}\right)$ and the full lines to the FIPs ( $U_{1}$ and $U_{2}$ ). The results correspond to a given field amplitude $\mathcal{E}_{0}=0.015$ a.u. and a direction parallel to the internuclear axis. As the dipole coupling $\mu_{12}$ increases linearly with the internuclear distance, the dissociative state $V_{2}$ becomes bounded $\left(U_{2}\right)$, while the bounded state $V_{1}$ becomes predissociative $\left(U_{1}\right)$. As a consequence, a wave packet in $U_{1}$ experiences bond softening, i.e., when the wave packet reaches the potential barrier, part of it leads to dissociation of $\mathrm{H}_{2}^{+}$, and in $U_{2}$ experiences bond hardening. The dressed electronic wave functions can be approximately obtained from the BornOppenheimer states by applying the rotation matrices obtained previously, as $\psi_{i}^{D S}\left(z ; R, \mathcal{E}_{0}\right)=\mathrm{W}_{i j}^{-1}\left(R, \mathcal{E}_{0}\right) \psi_{j}^{B O}(z ; R)$, where we use the sum index criteria. The approximation is only valid as long as very few electronic states participate, owing to the fact that practically only the lowest two electronic states determine the dynamics in the proposed set-up.

In our simulations, the dynamics is initiated in $V_{1}(R)$ with a wave packet that resembles the lowest vibrational wave function of the ground state of the $\mathrm{H}_{2}$ molecule, accounting for the process after the fast ionization of the neutral $\mathrm{H}_{2}$ molecule. Since $V_{1}(R)$ is very similar to $U_{1}(R)$ in the region of internuclear distances in which the wave packet is created, we will assume that the presence of the strong DC field does not change the initial wave function. In any case, the overall results are not affected by small variations on the shape of the initial wave function. An ultrashort pump pulse $\mathcal{E}_{p}(t)$ is then used to excite the wave packet to $U_{2}$, where it will remain trapped in the FIP. The classical turning point for the nuclear motion depends on the energy in $U_{2}$, which is a function of the time delay $t_{0}$ at which the pump pulse is turned on with respect to the time at which ionization takes place, and the amplitude of the field $\mathcal{E}_{0}$.

The process that we have qualitatively described so far shows how the action of a strong static field can be used to control the nuclear motion, essentially following a $\mathrm{LAMB}^{36,37}$ (Laser Adiabatic Manipulation of the Bond) 

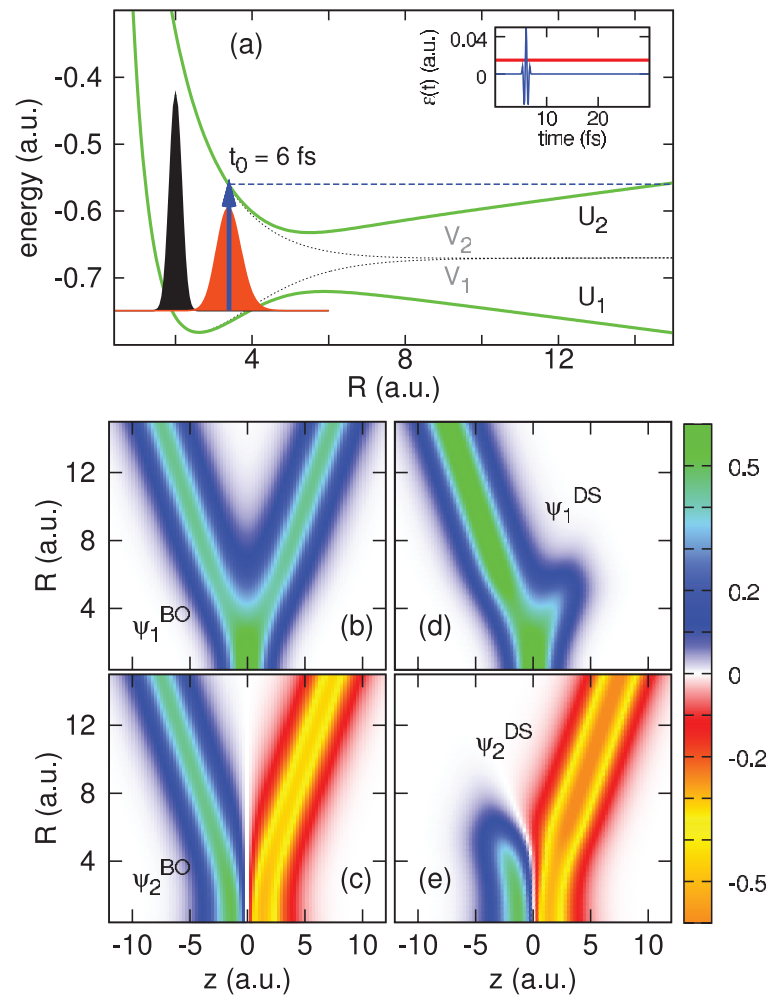

FIG. 1. (Upper panel) (a) Schematic representation of the first two electronic states of $\mathrm{H}_{2}^{+}$within the Born-Oppenheimer approximation (dotted lines) and the FIPs in the presence of a static field with $\mathcal{E}_{0}=0.0015$ a.u. (see text for details on the calculations of these states). The wave packet in black-full line represents a vertical transition from the ground state of the neutral hydrogen molecule, which freely evolves in the FIP associated to the ground state of $\mathrm{H}_{2}^{+}$. After a given delay, $t_{0}=6 \mathrm{fs}$, the wave packet (in orange solid line) has reached as far as $\mathrm{R} \approx 3.5$ a.u. At $t_{0}$, a 2 -fs pulse transfers most of the population to the FIP associated to the excited electronic state where the new wave packet remains trapped. The inset shows the field of the ultrashort pump pulse (blue line) and the DC field (red line) used in the above control scheme. (Lower panels) Electronic wave function as a function of electron $(z)$ and nuclear $(R)$ coordinates associated to (b) the BO ground state $\left(1 \mathrm{~s} \sigma_{g}\right)$, (c) the BO first excited state $\left(2 \mathrm{p} \sigma_{u}\right)$, (d) the field-dressed $1 \mathrm{~s} \sigma_{g}$ state, and (e) the field-dressed $2 \mathrm{p} \sigma_{u}$ state of $\mathrm{H}_{2}^{+}$.

scheme. In LAMB, the system is excited into a dissociative electronic state by an ultrashort (or highly chirped) pulse, after this state is stabilized by a "dressing" nonresonant strong field that creates the light-induced potential where the nuclear wave packet will evolve while the dressing pulse is switched on.

However, it is even more interesting to see how the electron motion proceeds in parallel. Figure 1 shows that $\psi_{1}^{B O}(z ; R)$ and $\psi_{2}^{B O}(z ; R)$ look like harmonic oscillator eigenstates when $R$ is short, and become degenerate, like symmetric eigenstates of a double well potential, when $R$ is large. This implies that, for these BO states, the average position of the electron is always $\langle z(t)\rangle=0$, that is, there is always the same probability of finding the electron around each nuclei when the molecule dissociates. But, as mentioned above, in the presence of the strong static field, one should better look at the dressed electronic states $\psi_{1}^{D S}\left(z ; R, \mathcal{E}_{0}\right)$ and $\psi_{2}^{D S}\left(z ; R, \mathcal{E}_{0}\right)$. These are also shown in Fig. 1. As can be seen, the field breaks the original symmetry of $\psi_{j}^{B O}(z ; R)$ states, leading to privileged directions. Depending on the FIP, the electron will move to $+z$ or $-z$ as the protons separate, that is, the electron will end in one proton or the other as the bond breaks. Under a positive field $\mathcal{E}_{0}$, bound electronic wave functions with even parity imply electron displacement along the gradient of the field as $R$ increases (i.e., $-z$ ), while in odd parity electronic wave functions, the electron displaces against the gradient $(+z)$.

\section{DYNAMICS AND CONTROL OF THE ELECTRONIC DIPOLE}

In Fig. 2, we show results of the actual dynamics obtained by solving Eq. (1) with $\mathcal{E}(t)=\mathcal{E}_{p}(t)+\mathcal{E}_{0}$. For the simulation shown in Fig. 2, we have chosen a DC field of $\mathcal{E}_{0}$ $=0.015$ a.u. The ultrashort pump pulse $\mathcal{E}_{p}(t)=\mathcal{E}_{p} \cos ^{2}$ $\left(\pi\left(t-t_{0}\right) / \tau\right) \cos \left(\omega_{p}\left(t-t_{0}\right)\right)$ (for $\left.-\tau / 2 \leq t-t_{0} \leq \tau / 2\right)$ of $\tau$ $=2 \mathrm{fs}$ duration and carrier frequency $\omega_{p}=5.4 \mathrm{eV}$, with peak amplitude of $\mathcal{E}_{p}=0.05$ a.u. is switched on such that it reaches its maximum at $t_{0}=6 \mathrm{fs}$. The duration, frequency, and intensity are chosen to maximize population transfer from $U_{1}$ to $U_{2}$ at the Franck-Condon window given by the wave packet position after $t_{0}$. With this choice, the population in $U_{2}$ just after the pulse is $\sim 0.7$, which remains practically constant
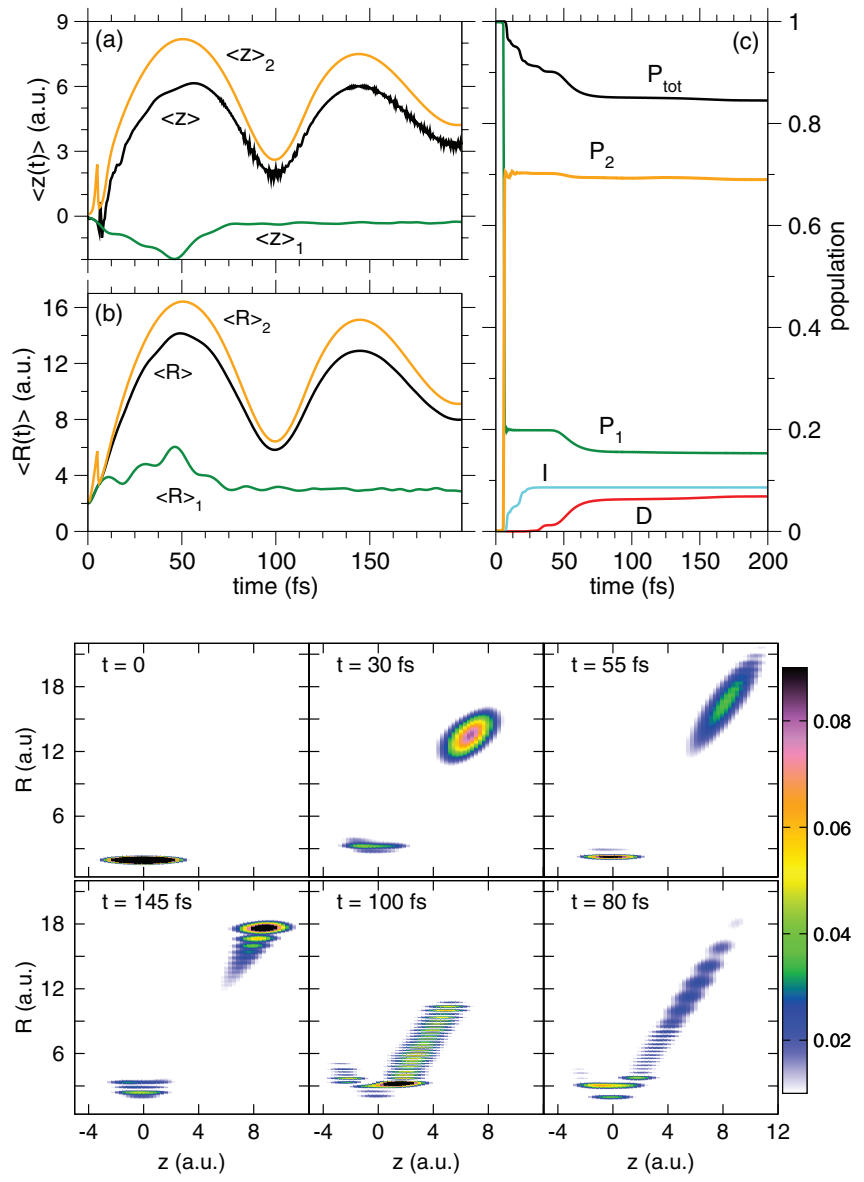

FIG. 2. (Upper panels) (a) Average electron position and (b) average internuclear distance as functions of time. (c) Probability as a function of time for dissociation (D), ionization (I), and the population remaining in the $U_{1}$ and $U_{2}$ FIPs $\left(\mathrm{P}_{1}\right.$ and $\mathrm{P}_{2}$, respectively). Notice that the average electron position, as defined in Eq. (6), is identical to the dipole moment of the molecule in the center of mass frame when multiplied by the electron charge. (Lower panels) Snapshots of the probability density as a function of electron and (inter)nuclear position at six different times. 
after the first few femtoseconds. The ionization probability for $\mathcal{E}_{0}=0.015$ a.u. is small $\left(P_{\text {ion }}<0.1\right)$. We note that ionization starts to be visible well after the pulse is gone. This is because the ionization probability from the $\psi_{2}^{B O}$ state (alternatively $\left.\psi_{2}^{D S}\right)$ is much larger than from the $\psi_{1}^{B O}$ state $\left(\psi_{1}^{D S}\right)$, as the former state is closer to the ionization limit. The probability of dissociation is also small $\left(P_{d i s} \approx 0.07\right)$. For more intense DC fields $\left(\mathcal{E}_{0}>0.04\right.$ a.u. $)$, the ionization probability increases. The same occurs for the dissociation probability, as the wave packet remaining in $U_{1}$ can dissociate via bond softening.

Figure 2 also shows the average electron position and internuclear distance

$$
\begin{aligned}
\langle z(t)\rangle & =\langle\psi(z, R, t)|z| \psi(z, R, t)\rangle_{z, R}, \\
\langle R(t)\rangle & =\langle\psi(z, R, t)|R| \psi(z, R, t)\rangle_{z, R},
\end{aligned}
$$

where we integrate over both the electron and nuclear coordinates. To better visualize the control process, which takes place in $U_{2}$, it is also useful to calculate the average positions projection onto the dressed electronic states. Defining $\phi_{j}(R, t)$ as the adiabatic nuclear wave function in the FIP $U_{j}$,

$$
\phi_{j}(R, t)=\left\langle\psi_{j}^{D S}\left(z ; R, \mathcal{E}_{0}\right) \mid \psi(z, R, t)\right\rangle_{z},
$$

the corresponding averaged projected positions are obtained as

$$
\begin{gathered}
\langle R(t)\rangle_{j}=\left\langle\phi_{j}(R, t)|R| \phi_{j}(R, t)\right\rangle_{R}, \\
\langle z(t)\rangle_{j}=\left\langle\phi_{j}(R, t) \psi_{j}^{D S}\left(z ; R, \mathcal{E}_{0}\right)|z| \phi_{j}(R, t) \psi_{j}^{D S}\left(z ; R, \mathcal{E}_{0}\right)\right\rangle_{z, R} .
\end{gathered}
$$

A similar calculation can be performed to obtain the average positions projection onto the Born-Oppenheimer states. While $\langle R(t)\rangle_{j}$ are very similar using $\psi_{j}^{D S}\left(z ; R, \mathcal{E}_{0}\right)$ and $\psi_{j}^{B O}(z ; R)$ (another consequence of the dynamics being mainly driven on a single FIP), the symmetry breaking of the electronic motion (into positive or negative $z$ ) can only be observed using the dressed states basis and thus we only show the results for the average positions projection onto the FIPs. Clearly, the parts of the wave packet remaining in $U_{1}$ reduce a bit $\langle R\rangle$ and $\langle z\rangle$. For the latter, one should remember that the electron moves in opposite directions in $U_{1}$ and $U_{2}$.

Figure 2 shows how the motion of the electron and protons is clearly correlated, with the electron always staying in between the two protons, but mainly leaving with the one at positive $z$ as the protons move apart. Thus the period of both motions is practically the same. The correlation between the electronic and nuclear motion is also clearly revealed in snapshots of the two-dimensional time-dependent density $\left|\psi(z, R, t)^{2}\right|$ as shown in the lower panel of Fig. 2. As can be seen, the wave packet moves over a long distance both in the $z$ and $R$ coordinates, where the FIP is very anharmonic and consequently dephasing makes the wave packet to quickly spread. Thus the maxima and minima of $\langle R(t)\rangle$ and $\langle z(t)\rangle$ become less pronounced after a few periods. For weak DC fields (and weaker bonds), there can be as few as 2 periods, whereas for stronger fields one can easily observe 10 periods of mo- tion. In principle, at larger times one could expect the revival of the periodic motion.

The ability to control the amplitude and specially the period of the dipole by manipulating the strength of the DC field $\mathcal{E}_{0}$ and the time $t_{0}$ at which the pump pulse is turned on, would give this control scheme very interesting properties, as the frequency of the emitting dipole (and its intensity via the amplitude of the motion) would be also controlled. Obviously, the carrier frequency of the pump pulse should also be adjusted, depending on the previous parameters, in order to maximize population transfer from $U_{1}(R)$ to $U_{2}(R)$ at time $t_{0}$. Clearly, as $t_{0}$ increases and the initial nuclear wave packet reaches larger bond distances before the pump pulse sets in, the energy difference between $U_{2}(R)$ and $U_{1}(R)$ at the Franck-Condon window (given by $t_{0}$ ) is smaller, so that one would expect that lower frequencies are required.

With this idea in mind, we have computed the amplitude of the dipole $\Delta z$ as the range of the dipole motion, $\left\langle z\left(t_{\text {max }}\right)\right\rangle$ $-\left\langle z\left(t_{\min }\right)\right\rangle$, where $t_{\max }$ is the time at which $\langle z\rangle$ reaches its first maximum and $t_{\min }$ is the time at which $\langle z\rangle$ is at a minimum during the first period. Similarly we calculate the bond elongation $\Delta R$ as the difference between the maxima and minima in $\langle R(t)\rangle$. In Fig. 3, we show how $\Delta z, \Delta R$, and the period of the dipole $T$ change as functions of $\mathcal{E}_{0}$ and $t_{0}$, together with the probabilities of dissociation (via bond softening from $U_{1}$ ) and ionization (predominantly from $U_{2}$ after the pump pulse has been turned on). As can be seen, the ionization and dissociation probabilities are small up to $\mathcal{E}_{0}=0.03$ a.u. Beyond $\mathcal{E}_{0}=0.05$ a.u. ionization and dissociation dominate and, consequently, the control scheme breaks down. For the range of parameters where the control scheme works, the period varies from $\sim 300$ fs (or more, for weaker fields than $\mathcal{E}_{0}$ $=0.005$ a.u.) down to 25 fs, i.e., over an order of magnitude. These correspond to frequencies in the far infrared, from 3 to $40 \mathrm{THz}$ approximately. For the lower frequencies, fewer periods of the electronic motion are observed before the wave packet disperses; the motion becomes more periodic as the frequency increases because the amplitude of the dipole is smaller.

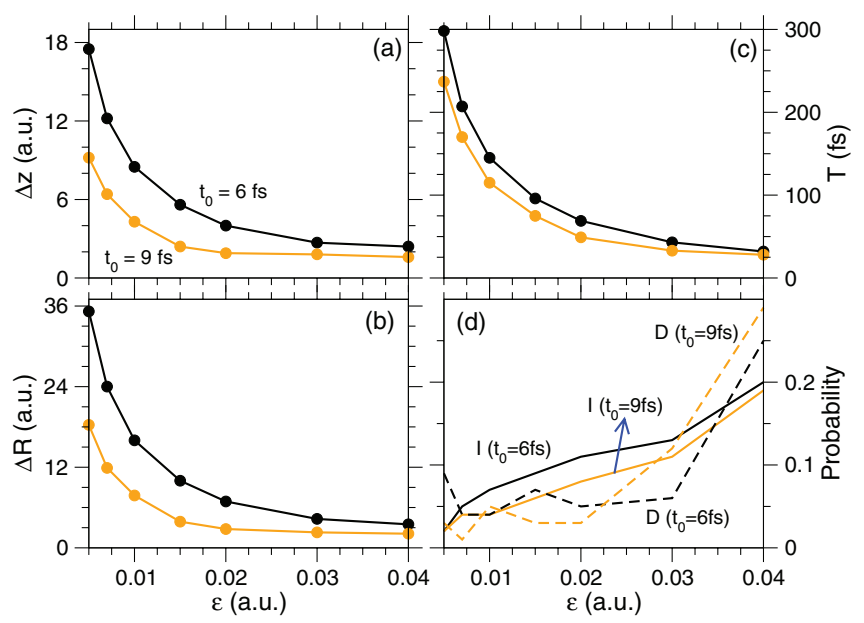

FIG. 3. (a) Amplitude of the dipole, (b) bond elongation, (c) dipole period, and (d) probabilities of dissociation and ionization as functions of $\mathcal{E}_{0}$ for different time-delays of the pump pulse $t_{0}$. 


\section{CONCLUSIONS}

In conclusion, we have proposed and numerically tested a laser scheme in combination with strong static electric fields to prepare huge oscillating dipoles whose amplitude and (slow) period of motion can be tailored as demanded. The amplitude of the dipole, of the order of 20-50 Debyes (or even larger for weaker electric fields), is 5 times larger than the largest known dipole in a diatomic molecule in the ground state (cesium chloride). Presently, several technical problems could limit the experimental implementation of this scheme in $\mathrm{H}_{2}^{+}$. First, the required frequency, duration, and intensity of the ultrashort $\mathrm{UV}$ pulse that is needed to pump $\mathrm{H}_{2}^{+}$lie at the edge of the available laser technology. Less demanding laser parameters would be necessary for diatomic cations with more favorable Franck-Condon transitions in the region of interest. Alternatively, one could first prepare the initial state in the parent $\mathrm{H}_{2}$ molecule by means of lasers, such that the wave packet before the ionization is closer to the desired FranckCondon region. The main limitation is related to the intensity of the DC field. Although the scheme works with weak DC fields as well as with far infrared laser pulses, the optimum conditions are found for a strong DC field, for which losses due to dissociation are minimal. Current technology sets $\mathcal{E}_{0} \sim 0.001$ a.u. as the maximum field that can be obtained in ice film capacitors, ${ }^{38}$ whereas for metal capacitors in vacuum the maximum value is even quite lower. These limits are still lower than the field amplitudes used in this work. However, the local field induced by an STM tip can be as large as 0.05 a.u. ${ }^{39}$ Although there are no simple ways of implementing these technologies to the proposed set-up with $\mathrm{H}_{2}^{+}$ or other cations, there are not known fundamental reasons to prevent static (local) fields to reach the required intensities in the near future. In any case, the proposed scheme shows how the coherent superposition of both electronic and nuclear states can yield highly correlated electron and nuclear motion that allows one to create, in the simplest possible molecule, the closest analog of a classical (macroscopic) dipole, thus opening the door to yet unseeing technological applications, e.g., novel sources of electromagnetic radiation.

\section{ACKNOWLEDGMENTS}

This work was supported by the NRF grant funded by the Korean government (2007-0056343 and 2012M3C1A6035358), the Basic Science Research program funded by MEST (2010-0005143), the Advanced Grant of the European Research Council XCHEM 290853, the European grant MC-RG ATTOTREND, the European COST Actions CM0702 (CUSPFEL) and CM1204 (XLIC), the European ITN CORINF, the MICINN Project Nos. CTQ2012-36184, FIS2010-15127, and CSD 2007-00010 (Spain), and the ERA-Chemistry project PIM2010EEC-00751.

\footnotetext{
${ }^{1}$ M. Hentschel, R. Kienberger, C. Spielmann, G. A. Reider, N. Milosevic, T. Brabec, P. Corkum, U. Heinzmann, M. Drescher, and F. Krausz, Nature (London) 414, 509 (2001)
}

${ }^{2}$ M. Drescher, M. Hentschel, R. Kienberger, M. Uiberacker, V. Yakovlev, A. Scrinzi, T. H. Westerwalbesloh, U. Kleineberg, U. Heinzmann, and F. Krausz, Nature (London) 419, 803 (2002).

${ }^{3}$ G. Sansone, E. Benedetti, F. Calegari, C. Vozzi, L. Avaldi, R. Flammini, L. Poletto, P. Villoresi, C. Altucci, R. Velotta, S. Stagira, S. De Silvestri, and M. Nisoli, Science 314, 443 (2006).

${ }^{4}$ J. M. Hutson, Science 327, 788 (2010).

${ }^{5}$ L. Santos, G. V. Shlyapnikov, P. Zoller, and M. Lewenstein, Phys. Rev. Lett. 85, 1791 (2000).

${ }^{6}$ K.-K. Ni, S. Ospelkaus, M. H. G. de Miranda, A. Pe'er, B. Neyenhuis, J. J. Zirbel, S. Kotochigova, P. S. Julienne, D. S. Jin, and J. Ye, Science 322, 231 (2008).

${ }^{7}$ S. Ospelkaus, K.-K. Ni, D. Wang, M. H. G. de Miranda, B. Neyenhuis, G. Quéméner, P. S. Julienne, J. L. Bohn, D. S. Jin, and J. Ye, Science 327, 853 (2010).

${ }^{8}$ W. Li, T. Pohl, J. M. Rost, S. T. Rittenhouse, H. R. Sadeghpour, J. Nipper, B. Butscher, J. B. Balewski, V. Bendkowsky, R. Löw, and T. Pfau, Science 334, 1110 (2011).

${ }^{9}$ H. Niikura, F. Légaré, R. Hasbani, A. D. Bandrauk, M. Y. Ivanov, D. M. Villeneuve, and P. B. Corkum, Nature (London) 417, 917 (2002).

${ }^{10}$ H. Niikura, F. Legare, R. Hasbani, M. Ivanov, D. Villeneuve, and P. Corkum, Nature (London) 421, 826 (2003).

${ }^{11}$ M. F. Kling, C. Siedschlag, A. J. Verhoef, J. I. Khan, M. Schultze, T. Uphues, Y. Ni, M. Uiberacker, M. Drescher, F. Krausz, and M. J. J. Vrakking, Science 312, 246 (2006).

${ }^{12}$ M. Kremer, B. Fischer, B. Feuerstein, V. L. B. de Jesus, V. Sharma, C. Hofrichter, A. Rudenko, U. Thumm, C. D. Schröter, R. Moshammer, and J. Ullrich, Phys. Rev. Lett. 103, 213003 (2009).

${ }^{13}$ T. Seideman, M. Y. Ivanov, and P. B. Corkum, Phys. Rev. Lett. 75, 2819 (1995).

${ }^{14}$ A. Conjusteau, A. D. Bandrauk, and P. B. Corkum, J. Chem. Phys. 106, 9095 (1997).

${ }^{15}$ B. Sheehy, B. Walker, and L. F. DiMauro, Phys. Rev. Lett. 74, 4799 (1995)

${ }^{16}$ E. Charron, A. Giusti-suzor, and F. H. Mies, Phys. Rev. Lett. 75, 2815 (1995).

${ }^{17}$ I. Franco and P. Brumer, J. Phys. B 41, 074003 (2008).

${ }^{18}$ C. Wunderlich, H. Figger, and T. W. Hänsch, Chem. Phys. Lett. 256, 43 (1996).

${ }^{19}$ H. Niikura, D. M. Villeneuve, and P. B. Corkum, Phys. Rev. Lett. 92, 133002 (2004).

${ }^{20}$ G. Yudin, S. Chelkowski, J. Itatani, A. Bandrauk, and P. Corkum, Phys. Rev. A 72, 051401(R) (2005).

${ }^{21}$ J. Javanainen, J. Eberly, and Q. Su, Phys. Rev. A 38, 3430 (1988).

${ }^{22}$ Q. Su and J. Eberly, Phys. Rev. A 44, 5997 (1991).

${ }^{23}$ K. C. Kulander, F. H. Mies, and K. J. Schafer, Phys. Rev. A 53, 2562 (1996).

${ }^{24}$ L. Roso, L. Plaja, P. Moreno, E. C. Jarque, J. R. V. de Aldana, J. San Roman, and C. Ruiz, Laser Phys. 15, 1393 (2005).

${ }^{25}$ M. D. Feit and J. A. Fleck, Appl. Opt. 17, 3990 (1978).

${ }^{26}$ R. Kosloff, J. Phys. Chem. 92, 2087 (1988).

${ }^{27}$ J.-M. Yuan and T. F. George, J. Chem. Phys. 68, 3040 (1978).

${ }^{28}$ A. D. Bandrauk and M. L. Sink, J. Chem. Phys. 74, 1110 (1981).

${ }^{29}$ S. W. Allendorf and A. Szoke, Phys. Rev. A 44, 518 (1991).

${ }^{30}$ A. Giusti-Suzor, F. H. Mies, L. F. Dimauro, E. Charron, and B. Yang, J. Phys. B 28, 309 (1995).

${ }^{31}$ A. Zavriyev, P. H. Bucksbaum, J. Squier, and F. Saline, Phys. Rev. Lett. 70, 1077 (1993).

${ }^{32}$ L. J. Frasinski, J. H. Posthumus, J. Plumridge, K. Codling, P. F. Taday, and A. J. Langley, Phys. Rev. Lett. 83, 3625 (1999).

${ }^{33}$ C. C. Marston and G. G. Balint-Kurti, J. Chem. Phys. 91, 3571 (1989).

${ }^{34}$ O. I. Tolstikhin, V. N. Ostrovsky, and H. Nakamura, Phys. Rev. Lett. 79, 2026 (1997).

${ }^{35}$ R. Santra, J. M. Shainline, and C. H. Greene, Phys. Rev. A 71, 032703 (2005).

${ }^{36}$ B. Y. Chang, H. Rabitz, and I. R. Sola, Phys. Rev. A 68, 031402 (2003).

${ }^{37}$ B. Y. Chang, S. Shin, and I. R. Sola, Phys. Rev. A 82, 063414 (2010).

${ }^{38} \mathrm{H}$. Kang, private communication (2013).

${ }^{39}$ M. Devel, C. Girard, C. Joachim, and D. J. F. Martin, Appl. Surf. Sci. 87-88, 390 (1995) 